\title{
Immediate Effect of the Complex Rotational Stretching Method on the Proprioceptive Sensation of the Shoulder Joint, Subacromial Space, Range of Motion, Shoulder Instability and Dynamic Function
}

\author{
Eun-Ho Lee · Hyo-Geun Kim · Dongyeop Lee, PT, PhD · Ji-Heon Hong, PT, PhD · \\ Jae-Ho Yu, PT, PhD · Jin-Seop Kim, PT, PhD · Seong-Gil Kim, PT, PhD ${ }^{\dagger}$
}

Department of Physical Therapy, College of Health Science, Sunmoon University

Received: December 92021 / Revised: December 152021 / Accepted: January 172022

(C) 2022 J Korean Soc Phys Med

\begin{abstract}
| Abstract |
PURPOSE: This study compared the effects of proprioceptive sensation, subacromial space, and dynamic function according to proprioceptive neuromuscular facilitation (PNF), static stretching (SS), and complex rotational stretching (CRS).
\end{abstract}

METHODS: Thirty students without any musculoskeletal disease who volunteered to participate were included in this study. The following metrics were measured to evaluate the function and stability under the normal conditions, with the PNF, SS, and CRS: special test and flexion, extension, abduction, adduction, internal rotation, external rotation (shoulder range of motion) and reaching distance on the medial (ME), superolateral (SL), inferolateral (IL), and subacromial space and proprioceptive sensation were evaluated. All measures were analyzed using one-way ANOVA and repeated measures of ANOVA.

RESULTS: A clear difference in adduction in the range of

$\uparrow$ Corresponding Author : Seong-Gil Kim sungkil83@naver.com, https://orcid.org/0000-0002-2487-5122 This is an Open Access article distributed under the terms of the Creative Commons Attribution Non-Commercial License (http://creativecommons.org/licenses/by-nc/3.0) which permits unrestricted non-commercial use, distribution, and reproduction in any medium, provided the original work is properly cited. motion was observed in all groups $(p<.05)$. Significant differences could not be identified in all values in the error test, except for $\mathrm{Ab}(\mathrm{p}<.05)$. Significant differences in reach were noted in all directions of the SS and PNF in the Me, SL, and IL $(p<.05)$. After the intervention, significant differences in the average values could be identified in all groups except for the SS group after rest $(p<.05)$. After the intervention, there was a significant difference between the CRS and SS and PNF groups $(\mathrm{p}<.05)$.

CONCLUSION: The application of CRS is as helpful as the existing SS and PNF for improving the joint range of motion improvement, shoulder balance, and subacromial space.

Key Words: Complex rotational stretching, Dynamic stability, PNF, Proprioception, Static stretching

\section{Introduction}

Movement of the body is a fundamental component of human life. People usually interact with the surrounding environment during most of their daily activities. In recent decades, an understanding of the role that sensory information plays in neuroplasticity has increased through 
mechanisms examining human motor control [1]. To control movement, the brain must integrate proprioceptive information from various mechanoreceptors. The role of proprioception in daily activities, exercise, and sports has been investigated extensively using various techniques, but the proprioceptive mechanisms underlying human movement control remain unclear [2].

In previous experiments, proprioception and understanding of the three test methods were reviewed. Passive motion detection, joint position reproduction, and active range of motion identification were used to evaluate proprioception [3]. Charles Sherrington defined proprioception as the sense that allows a person to know where any part of the body is at any time, as "the perception of joints and body movements as well as the position of a body or body segment in space". He also described proprioception as the "perception" of body position and movement. All perception requires signals within the nervous system derived from physical stimuli from various sensory organs. Therefore, proprioception requires the stimulation of mechanoreceptors to a threshold through body movement (change in body position) [4].

However, perception is formed not simply by the passive reception of sensory signals but by memory and learning. In this understanding, proprioception can be defined as an individual's ability to determine body segment position and spatial movement by integrating the sensory signals from mechanoreceptors [5]. As the energy-efficient movement patterns develop, they rely on passive structures for energy storage, muscle tissue structures for the generation and control of movement, and neurological control to coordinate smooth movements [6]. Stretching is used mainly therapeutically in clinical practice and includes dynamic stretching, static stretching (SS), and proprioceptive neuromuscular facilitation (PNF) [7]. According to Nelon (2006), SS was defined as extending a specific muscle to the farthest point possible and maintaining that position for a certain period. It is also used frequently for certain active warm-ups to improve performance. Moreover, it was reported to increase the joint range of motion by $2.8 \%$ in a specific population [8].

Although previous studies reported that PNF increases flexibility over SS, it is important to evaluate the increase in flexibility in athletes using both techniques. Given that SS is easier to perform, it is important to evaluate whether increasing flexibility with SS is comparable to PNF stretching compared to athletes who do not stretch. Thus, SS increased the ROM more than dynamic. This supports the use of SS within the activity-specific warm-up to ensure the maximum ROM [9]. In addition, fixed or PNF stretching can be used for athletes who want to increase their range of motion. This is important because there is more evidence that SS and PNF stretching increase the range of motion and that both stretching methods can be selected [10].

SS is a useful way to improve muscle flexibility and joint mobility. Moreover, it is an effective way to prevent joint contractures, reduce muscle tension and improve muscle flexibility. Several studies showed that the maximum ROM increased immediately after SS and that the passive torque or muscle-tendon unit stiffness decreased after SS [11]. SS has been used to improve the range of motion and injury prevention. Researchers examined the effect of SS on the shoulder ROM, especially the glenohumeral internal rotation ROM [12].

Disorders caused by stretching can affect the overall balance and stability or limb proprioception. On the other hand, there are no reports on the effect of acute stretching on balance and proprioception or response/motor time. Stretch-induced changes in proprioception or mechanical output are expected to affect the ability to adapt effectively to stability issues [13]. In addition, there are no studies investigating a program that utilizes a combination of proprioceptive balance exercises for dynamic posture control or studies examining the effect of an exercise program on dynamics [14].

Therefore, this study examined the effects of proprioception, 
Table 1. General Characteristics of the Participants

$(\mathrm{n}=30)$

\begin{tabular}{lccc}
\hline & CRS Ggroup $(\mathrm{n}=10)$ & SS Group $(\mathrm{n}=10)$ & PNF Group $(\mathrm{n}=10)$ \\
\hline Age (years) & 23.1 & 22 & 22.8 \\
Height $(\mathrm{cm})$ & 174.7 & 174.2 & 174.8 \\
Weight $(\mathrm{kg})$ & 73.5 & 72.5 & 71.2 \\
\hline
\end{tabular}

*Values indicate mean \pm standard deviation

joint range of motion, subacromial space, shoulder instability, and dynamic function according to proprioceptive neuromuscular stimulation, SS, and complex rotational stretching (CRS) methods before, after, and after rest.

This study hypothesized that the use of CRS has a similar effect to SS and PNF. This data can serve as a reference for comparison before and after exercise and for choosing an appropriate rest time.

\section{Methods}

\section{Participants}

This study was conducted on 30 healthy adults at $\mathrm{S}$ University in Asan, Chungcheongnam-do. The purpose and method of the study were fully explained to the subjects before participating. The subjects participated voluntarily in the study and met the selection criteria. The subject selection criteria for this study were those with no shoulder joint injury, no previous medical history, and no past orthopedic history of shoulder joint within three months. This study was approved by the Institutional Review Board (IRB) of Sunmoon University (SM-202104-023-1). All participants provided written informed consent and understood the purpose of the experiment and the study method. Before starting the experiment, height and weight were measured using an autonomic BMI measuring tadiometer (BSM 370, Korea, 2011). The number of people was assigned by random selection. Table 1 lists the characteristics of the participants.

\section{Measurements}

1) Range of motion of the Shoulder

This study compared the pre-post-rest in the three groups under CRS, PNF, or SS conditions. The flexion, extension, abduction, adduction, internal rotation, and external rotation of the shoulder joint were measured using a goniometer. All movements were measured in the sitting position, and the position of the goniometer was referred to as the axis. During shoulder flexion, attention was paid to the trunk, and the lumbar spine flexion, shoulder, abduction, and axis were set on the lateral part of the acromion. During the shoulder extension, attention was paid to the trunk, and spine flexion, abduction of the shoulder, and the axis were the same as for the shoulder flexion. For shoulder abduction, attention was paid to lateral bending of the trunk and shoulder flexion, and the axis was set in the anterior part of the acromion. During shoulder abduction, lateral bending of the trunk to the same side and depression of the scapular was prevented, and the axis was the same as shoulder abduction. The external and internal rotation of the shoulder starts with $90^{\circ}$ abduction of the shoulder joint and $90^{\circ}$ flexion of the elbow joint in a sitting position, and the axis is the elbow head of the ulna. During the external rotation of the shoulder, attention was paid to the trunk and lumbar flexion, scapula depression and elevation and elbow extension. The measurements were made three times for each movement, and the average value was analyzed.

2) Measuring stability of body (Dynamic Balance)

This study compared the pre-intervention, post-intervention, 
and rest between the three groups under PNF, SS, and CRS conditions. Before the measurement, the researcher explained the purpose and method of the experiment to the subject. The same environment was provided to all participants. The participants were instructed to wear the same short sleeves, and the measurement was conducted by the same researcher to reduce the error during the measurement. A physical examination was performed prior to functional testing to determine the stability. The dynamic stability was measured according to the distance reached during the Y-Balance test (Perform Better, U.S.A). The subject held one arm in the middle and pushed the block in the medial, inferolateral, and superolateral directions with the opposite arm. All subjects were barefoot at the time of measurement because shoes could affect stability. Subjects performed two exercises in each direction and then performed three measurements in each direction. The measurement was carried out with one arm supported, legs stretched back, and postures maintained in each direction. The maximum reach was measured by reading a tape measure on the edge of the reach indicator at the point where the farthest part of the arm reached [15]. In the event of failure to maintain posture on the platform (e.g., the outstretched arm touched the floor or fell off the stance platform or failed to maintain contact between the outstretched arm and the block), the round was discarded and re-measured. The formula to express the normalized numerical value is shown in Step 1 [6]. For the YBT distance calculation, the reach distances in the medial, inferolateral, and superolateral directions in Groupl before and after applying PNF, Group 2 before and after applying SS after resting, and Group 3 were compared before and after CRS application and after rest.

\section{3) Measuring arm length}

The length of the arm was measured in the anatomical position. The length of the subject's right arm in the anatomical position was then measured in centimeters from the scapular acromion to the most prominent part of the radius styloid process using a cloth tape measure.

\section{4) Measuring Subacromial space}

This study compared the pre-post-rest among the three groups under PNF, SS, or CRS conditions. Subacromial space was measured at $4-12 \mathrm{MHz}$ intensity in B mode of Ultrasonography (Ezono 3000, Germany, 2011). The participants were instructed to sit on a chair with their feet on a flat floor and capture an image after ultrasound measurement in a neutral torso posture, with the head straight and arms parallel to the neutral trunk posture. An ultrasound transducer was placed anteriorly to the edge of the anterior peak, as confirmed by palpation. The long axis of the transducer was placed in the plane of the scapula and parallel to the flat surface of the peak. The humerus head and peak were photographed to capture the anterior aspect of the subapical space, and the elongated length of this area was measured. The acromiohumeral distance (AHD) was defined as the two-dimensional shortest linear distance between the anterior-lower end of the peak and the head of the humerus. ADH uses the screen calipers of the ultrasound machine to visually locate the upper surface of the humerus head and the underside of the peaks and then measure the linear distance. The average of the $\mathrm{ADH}$ measurements of each of the two images found was used for data analysis. The intra-rater test-retest reliability of this $\mathrm{ADH}$ method showed good reliability and acceptable measurement error $(\mathrm{ICC} 3,2=.98, \mathrm{MDC} 90=.8 \mathrm{~mm})[16]$.

5) Measuring proprioceptive of Shoulder (Error test)

This study compared the pre-post-rest in the three groups under PNF, SS, or CRS conditions. With the laser pointer on the participant's hand, they were asked to stand upright and maintain a straightforward position with a neutral and comfortable arm position (Fig. 1). In the standing condition, the shoulder joint $90^{\circ}$ flexion and shoulder abduction $90^{\circ}$ abduction motion were performed. They were then asked 


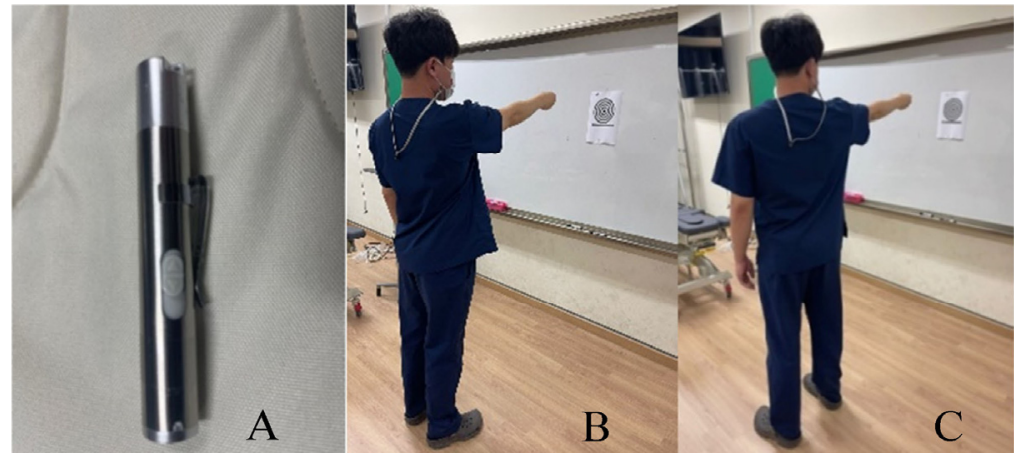

Fig. 1. Error test.

to change the positions slowly and verbally state the recognized position when performing the movement. One of the two researchers then used a pen to mark the end positions of all movements (flexion, abduction) of the subject. The average value was calculated by measuring the deviation of the end position mark for each repetition of each motion; the larger the error, the lower the proprioceptive sense [17].

\section{Experiment procedures}

CRS is a stretching that proceeds by controlling the compensation action of the other joints while feeling like drawing a circle while reaching the maximum range of all movements. In the order of motion, flexion, vowel, and outer rotation were the same: raising the upper arm to 12 o'clock, and returning to the anatomical posture while simultaneously proceeding with the temple, opening, and inner rotation. This is a stretch from the United States with a controlled artistic rotations full name called CARs. It is a stretch in which all movements reach the maximum range with a feeling of circulation while controlling the compensation action of other joints. Generally, when the joint is injured, the injury does not occur at a normal joint angle but when out of the maximum range. Therefore, a stretch is made to give balance and stability within the maximum range.

SS is a method of stretching without elasticity in a static state. This study performed stretching using six movements: bending, stretching, spreading, vowels, lateral turning, and inner turning.

PNF was conducted using the maintenance-relaxation technique. The motion used upper limb D1, D2 bending, and stretching, which are patterns of intrinsic water-soluble neuromuscular stimulation. Upper limb D1 bending is shoulder joint bending, vowel, lateral turn, front up of shoulder bone, back of front arm, wrist joint bending, no side up, finger bending, and vowel. Upper limb D2 bending is shoulder joint bending, extension, back of shoulder bone, wrist joint, extension, wrist joint. D1 and D2 deflection operations become deflection operations when the opposite operation of the bending operations shown above is performed. The number of such stretching is performed in 10 sets for 15 seconds, and the break time is 10 seconds.

\section{Statistical Analyses}

All statistical analyses in this experiment were performed using SPSS statistical software (version 20.0; IBM) program to calculate the mean and standard deviation for each measurement item. After normality verification, one-way ANOVA was used to compare the groups, and repeated measures of ANOVA were used to compare the changes before, immediately after, and five minutes after exercise for each exercise. A Fisher's least significant difference (LSD) test was performed for post-hoc analysis. 
Table 2. Differences among the Groups

\begin{tabular}{|c|c|c|c|c|c|c|}
\hline \multirow[b]{2}{*}{ variable } & \multirow[b]{2}{*}{ time } & \multicolumn{3}{|c|}{ Intervention } & \multicolumn{2}{|c|}{$(\mathrm{n}=30)$} \\
\hline & & CRS & SS & PNF & $\mathrm{F}$ & $\mathrm{p}$ \\
\hline \multirow[t]{3}{*}{ ROM-Fx } & Pre & 166.14 & 171.99 & $170.34 \pm 10.8$ & .963 & .391 \\
\hline & Post & 175.12 & 176.26 & $174.08 \pm 6.72$ & .173 & .840 \\
\hline & Follow-up & 174.3 & $175.12 \pm 11.33$ & $177.62 \pm 6.14$ & .368 & .695 \\
\hline \multirow[t]{3}{*}{ ROM-Ex } & Pre & $51.98 \pm 17.65$ & $45.81 \pm 11.44$ & $48.88 \pm 8.82$ & .541 & .587 \\
\hline & Post & $55 \pm 17.55$ & $49.37 \pm 9.33$ & $51.23 \pm 6.95$ & .556 & .582 \\
\hline & Follow-up & $52.9 \pm 12.27$ & $49.64 \pm 10.02$ & $50.74 \pm 7.37$ & .272 & .760 \\
\hline \multirow[t]{3}{*}{ ROM-Ab } & Pre & $160.84 \pm 14.78$ & $167.86 \pm 14.2$ & $167.98 \pm 13.85$ & .819 & .457 \\
\hline & Post & $167.75 \pm 19.26$ & $172.11 \pm 15.89$ & $172.8 \pm 9.73$ & .316 & .737 \\
\hline & Follow-up & $166.85 \pm 18.3$ & $173.89 \pm 16.3$ & $173.28 \pm 10.61$ & .644 & .533 \\
\hline \multirow[t]{3}{*}{ ROM-Ad } & Pre & $44.1 \pm 12.78$ & $35.38 \pm 8.78$ & $30.82 \pm 9.35$ & 4.164 & $.020 * 13$ \\
\hline & Post & $55.2 \pm 20.71$ & $45.2 \pm 14.39$ & $36.11 \pm 8.55$ & 3.859 & $.033 * 13$ \\
\hline & Follow-up & $50.51 \pm 16.32$ & $45.22 \pm 15.86$ & $37.5 \pm 7.7$ & 2.221 & .122 \\
\hline \multirow[t]{3}{*}{ ROM-ER } & Pre & $96.63 \pm 15.11$ & $104.44 \pm 7.61$ & $102.12 \pm 7.71$ & 1.435 & .253 \\
\hline & Post & $101.66 \pm 16.31$ & $107.65 \pm 11.08$ & $109.44 \pm 7.26$ & 1.126 & .331 \\
\hline & Follow-up & $104.24 \pm 17.91$ & $110.26 \pm 5.76$ & $110.26 \pm 11.36$ & .754 & .488 \\
\hline \multicolumn{6}{|l|}{ ROM-IR } & .236 \\
\hline & Post & $67.74 \pm 19.61$ & $64.76 \pm 21.08$ & $53.3 \pm 9.09$ & 1.914 & .163 \\
\hline \multirow{3}{*}{ ET-Fx } & Follow-up & $64.22 \pm 20.29$ & $64.07 \pm 19.28$ & $52.72 \pm 10.86$ & 1.442 & .256 \\
\hline & Pre & $8.63 \pm 6.11$ & $9.65 \pm 3.92$ & $7.17 \pm 3.09$ & .742 & .485 \\
\hline & Post & $9.81 \pm 5.48$ & $7.01 \pm 7.04$ & $5.91 \pm 5.06$ & 1.158 & .330 \\
\hline \multirow{3}{*}{ ET-Ab } & Follow-up & $7.87 \pm 1.54$ & $6.27 \pm 2.44$ & $6.03 \pm 3.88$ & 1.275 & .298 \\
\hline & Pre & $8.94 \pm 4.27$ & $12.15 \pm 6.91$ & $13.1 \pm 5.05$ & 1.551 & .234 \\
\hline & Post & $8.23 \pm 3.03$ & $8.86 \pm 3.8$ & $6.81 \pm 2.28$ & 1.145 & .338 \\
\hline \multirow[t]{3}{*}{$\mathrm{Me}$} & Follow-up & $11.6 \pm 7.3$ & $8.9 \pm 4.74$ & $6.34 \pm 3.11$ & 2.429 & .108 \\
\hline & Pre & $54.1 \pm 6.79$ & $53.4 \pm 4.02$ & $55.92 \pm 12.57$ & .236 & .793 \\
\hline & Post & $59.86 \pm 6.68$ & $57.34 \pm 4.23$ & $61.47 \pm 12.82$ & .563 & .570 \\
\hline \multirow[t]{3}{*}{ SL } & Follow-up & $59.76 \pm 6.62$ & $59.76 \pm 6.62$ & $59.65 \pm 11.57$ & .225 & .796 \\
\hline & Pre & $67.84 \pm 9.3$ & $63.73 \pm 9.12$ & $65.9 \pm 11.24$ & .423 & .653 \\
\hline & Post & $71.57 \pm 8.95$ & $71.94 \pm 5.83$ & $70.72 \pm 10.82$ & .056 & .954 \\
\hline IL & Follow-up & $70.7 \pm 9.97$ & $70.93 \pm 6.75$ & $68.83 \pm 12.61$ & .139 & .872 \\
\hline \multirow{6}{*}{ SONO } & Pre & $88.4 \pm 11.15$ & $94.66 \pm 14.44$ & $90.89 \pm 7.53$ & .766 & .476 \\
\hline & Post & $91.64 \pm 12.04$ & $104.05 \pm 12.19$ & $96.4 \pm 11.69$ & 2.731 & .088 \\
\hline & Follow-up & $91.19 \pm 12.77$ & $101.37 \pm 13.17$ & $93.47 \pm 11.53$ & 1.826 & .181 \\
\hline & Pre & $8.61 \pm 2.6$ & $10.49 \pm 2.39$ & $11.54 \pm 1.85$ & 4.146 & $.020^{* 13}$ \\
\hline & Post & $9.71 \pm 2.55$ & $11.38 \pm 2.24$ & $13.43 \pm 2.71$ & 5.501 & $.011^{* 13}$ \\
\hline & Follow-up & $10.66 \pm 2.28$ & $10.93 \pm 3.38$ & $12.75 \pm 2.22$ & 1.794 & .182 \\
\hline
\end{tabular}

${ }^{*} \mathrm{p}<.05$ (Mean \pm SD), CRS: complex rotational stretching group, SS: static stretching group, PNF: proprioceptive neuromuscular facilitation group ROM: range of motion ET: error test, SONO: sonography, (Fx, Flxion), (Ex, Extension), (Ab, Abduction), (Ad, Adduction), (ER, External rotation), (IR, Internal rotation), (ME, Medial), (SL, Superiolateral), (IL, Inferiolateral), $\left({ }^{1}, \mathrm{CRS}\right),\left({ }^{2}, \mathrm{SS}\right),\left({ }^{3}, \mathrm{PNF}\right)$ 
The statistical significance level was set to $\mathrm{p}<.05$.

\section{Results}

\section{Compare differences among group}

There was no significant difference in shoulder joint range of motion angle values in flexion, extension, abduction, external rotation, and internal rotation between the CRS, SS, and PNF groups ( $p>$.05). Significant differences in the angular shoulder joint range of motion in the vowel posture were found before and after the intervention between the CRS, SS, and PNF groups $(\mathrm{P}<.05)$.

In the error test, the distance from the central point was compared between groups to measure the proprioceptive sensation of $90^{\circ}$ bending and abduction motions. In the error test, the postures of flexion and abduction were similar in the CRS, SS, and PNF groups ( $\mathrm{P}>.05)$.

The dynamic balance ability was compared in groups by comparing the reaching distance values in the inner, upper and lower directions. No significant differences were found in the medial and lateral directions between the CRS, SS, and PNF groups ( $>$.05). Significant differences in the downward direction were found between the CRS, SS, and PNF groups after the intervention except before and after rest $(\mathrm{P}<.05)$.

Ultrasound was used to compare the mean subacromial space between the groups. Significant differences were observed between the CRS, SS, and PNF groups before and after the intervention except after rest $(\mathrm{P}<.05)$.

[Table 2] shows the results of post-hoc analysis. Significant differences in vowel motion in the shoulder joint range of motion were found between the CRS and PNF groups before and after the intervention $(p<.05)$. In the dynamic balance ability, there was a significant difference between the CRS and SS after the downward direction intervention $(\mathrm{p}<.05)$. Ultrasound showed a significant difference in the CRS and PNF of the two groups before and after the intervention $(p<.05)$.
2. Compare pre- and post-status, Follow-up in each group

After the intervention, a significant difference in the range of motion was confirmed in the bending direction of the other groups except for the PNF group $(\mathrm{p}<.05)$. After the intervention was applied, a significant difference in the range of motion was noted in the abduction direction of the PNF group $(\mathrm{p}<.05)$. After the intervention, a significant difference in the range of motion was noted in the vowel direction of all groups $(p<.05)$. After the intervention was applied, a significant difference in the range of motion of lateral rotation was observed in all groups except for the SS group $(\mathrm{p}<.05)$. Except for this, there were no significant differences in the angle values in each direction $(p>.05)$.

The error test looked at the distance closer to the center point to measure the proprioceptive sensation of $90^{\circ}$ of bending and abduction motions. After the intervention, a significant difference was observed only in the divergence of the PNF group after the intervention $(p<.05)$. On the other hand, no significant difference was observed in any of the other values $(p>.05)$.

The dynamic balancing ability examined the reach value in the inward, upward and downward directions. After the intervention, significant differences in the reach distance were confirmed in the medial direction except for the upper and lower sides of the CRS group $(\mathrm{p}<.05)$. After the intervention, significant differences in reach were confirmed in all directions in the medial, upper, and lower directions of the SS and PNF groups $(\mathrm{p}<.05)$.

Ultrasound measured the length of the subacromial space. After the intervention was applied, a significant difference in the mean value was confirmed in all groups except for the SS group $(\mathrm{p}<.05)$.

Post-hoc analysis was performed as a subscript in [Table 3]. Significant differences were observed in the shoulder joint range of motion after intervention and rest in the CRS and SS groups of bending motion $(\mathrm{p}<.05)$. In the abduction 
I J Korean Soc Phys Med Vol. 17, No. 1

Table 3. Pre- and Post-status, Follow-up in Each Group

$(n=30)$

\begin{tabular}{|c|c|c|c|c|c|c|}
\hline Variable & Group & Pre & Post & Follow-up & $\mathrm{F}$ & $\mathrm{p}$ \\
\hline \multirow[t]{3}{*}{ ROM-Fx } & CRS & 166.14 & 175.12 & 174.3 & 6.223 & $.000^{* 12,13}$ \\
\hline & SS & 171.99 & 176.26 & 175.12 & 8.209 & $.000^{* 12,13}$ \\
\hline & PNF & 170.34 & 174.08 & 177.62 & 3.483 & .057 \\
\hline \multirow[t]{3}{*}{ ROM-Ex } & CRS & 51.98 & 55 & 52.9 & .406 & .671 \\
\hline & SS & 45.81 & 49.37 & 49.64 & 1.237 & .318 \\
\hline & PNF & 48.88 & 51.23 & 50.74 & .764 & .471 \\
\hline \multirow[t]{3}{*}{$\mathrm{ROM}-\mathrm{Ab}$} & CRS & 160.84 & 167.75 & 166.85 & 1.183 & .326 \\
\hline & SS & 167.86 & 172.11 & 173.89 & 2.651 & .090 \\
\hline & PNF & 167.98 & 172.8 & 173.28 & 5.871 & $.013^{* 12,13}$ \\
\hline \multirow[t]{3}{*}{ ROM-Ad } & CRS & 44.1 & 55.2 & 50.51 & 5.084 & $.011^{* 12,13}$ \\
\hline & SS & 35.38 & 45.2 & 45.22 & 7.944 & $.001^{* 12,13}$ \\
\hline & PNF & 30.82 & 36.11 & 37.5 & 13.875 & $.000^{* 12,13}$ \\
\hline \multirow[t]{3}{*}{ ROM-ER } & CRS & 96.63 & 101.66 & 104.24 & 4.398 & $.023^{* 12,13}$ \\
\hline & SS & 104.44 & 107.65 & 110.26 & 1.889 & .181 \\
\hline & PNF & 102.12 & 109.44 & 110.26 & 9.034 & $.000^{* 12,13}$ \\
\hline \multirow[t]{3}{*}{ ROM-IR } & CRS & 62.4 & 67.74 & 64.22 & 1.351 & .289 \\
\hline & SS & 59.03 & 64.76 & 64.07 & 3.181 & .067 \\
\hline & PNF & 49.51 & 53.3 & 52.72 & 1.023 & .378 \\
\hline \multirow[t]{3}{*}{ ET-Fx } & CRS & 8.63 & 9.81 & 7.87 & 1.658 & .253 \\
\hline & SS & 9.65 & 7.01 & 6.27 & 1.526 & .241 \\
\hline & PNF & 7.17 & 5.91 & 6.03 & .679 & .524 \\
\hline \multirow[t]{3}{*}{ ET-Ab } & CRS & 8.94 & 8.23 & 11.6 & 1.201 & .327 \\
\hline & SS & 12.15 & 8.86 & 8.9 & 3.581 & .494 \\
\hline & PNF & 13.1 & 6.81 & 6.34 & 5.551 & $.032^{* 12,13}$ \\
\hline \multirow[t]{3}{*}{$\mathrm{ME}$} & CRS & 54.1 & 59.86 & 59.76 & 7.457 & $.000^{* 12,13}$ \\
\hline & SS & 53.4 & 57.34 & 57.57 & 6.395 & $.004^{* 12,13}$ \\
\hline & PNF & 55.92 & 61.47 & 59.65 & 15.926 & $.000^{* 12,13}$ \\
\hline \multirow[t]{3}{*}{ SL } & CRS & 67.84 & 71.57 & 70.7 & 3.225 & .067 \\
\hline & SS & 63.73 & 71.94 & 70.93 & 4.915 & $.043^{* 12,13}$ \\
\hline & PNF & 65.9 & 70.72 & 68.83 & 5.061 & $.019^{* 12}$ \\
\hline \multirow[t]{3}{*}{ IL } & CRS & 88.4 & 91.64 & 91.19 & 1.266 & .306 \\
\hline & SS & 94.66 & 104.05 & 101.37 & 9.279 & $.000^{* 12,13}$ \\
\hline & PNF & 90.89 & 96.4 & 93.47 & 3.555 & $.050^{* 12}$ \\
\hline \multirow[t]{3}{*}{ SONO } & CRS & 8.61 & 9.71 & 10.66 & 12.306 & $.000^{* 13}$ \\
\hline & SS & 10.49 & 11.38 & 10.93 & .661 & .526 \\
\hline & PNF & 11.54 & 13.43 & 12.75 & 6.493 & $.000^{* 12,13}$ \\
\hline
\end{tabular}

${ }^{*} \mathrm{p}<.05$ (Mean \pm SD), CRS: complex rotational stretching group, SS: static stretching group, PNF: proprioceptive neuromuscular facilitation group ROM: range of motion ET: error test, SONO: sonography, (Fx, Flxion), (Ex, Extension), (Ab, Abduction), (Ad, Adduction), (ER, External rotation), (IR, Internal rotation), (ME, Medial), (SL, Superiolateral), (IL, Inferiolateral), ( ${ }^{1}$, pre), $\left({ }^{2}\right.$, post), $\left({ }^{3}\right.$, follow-up) 
movement, only the PNF group showed a significant difference after the intervention and after rest $(\mathrm{p}<.05)$. After the intervention of the CRS and PNF groups in vowel motion, there was a significant difference after rest $(\mathrm{p}<$ .05). After the intervention in the CRS and PNF groups, there was a significant difference in the lateral rotation movement after rest.

In the error test, a significant difference was observed in the abduction motion of the PNF group after the intervention and after the intervention and after rest $(\mathrm{p}<$ $.05)$. On the other hand, no other significant difference was found $(\mathrm{p}>.05)$.

In dynamic balance ability, significant differences in the medial direction were found in all groups after intervention and after rest $(\mathrm{p}<.05)$. In the upper-gastric side, a significant difference was noted after the intervention and after rest in SS, but a significant difference could be seen only after the intervention in PNF $(\mathrm{p}<.05)$. In SS, a significant difference was observed after the intervention and rest, but a significant difference was found only after intervention in PNF $(p<.05)$. Ultrasound revealed a significant difference only after intervention in the CRS group, but significant differences were noted in the PNF group after the intervention and rest $(p<.05)$.

\section{Discussion}

This study compared the effects of proprioception, joint range of motion, subacromial space, shoulder instability, and dynamic function according to the CRS, SS, and PNF before, after, and after rest. The study was conducted on 30 healthy people. As a result, there was a significant difference in the joint range of motion and ultrasound measurement for each group. Before starting the study, there was no significant difference between the three groups in the range of motion, error test, dynamic balance, and ultrasound test. Although SS and CRS did not significantly affect the joint range of motion, but PNF showed differences in various movements. In the dynamic balance, the CRS group showed a significant difference only in the ME direction. The SS and PNF groups showed a significant difference in all directions. A before-and-after difference was noted in the PNF. SS showed a significant difference in the two-way comparison between before, after, and after rest. During the ultrasound measurement, the CRS group had an improved score, but the effect was minimal compared to the PNF group. There was a difference in all bilateral comparisons in the PNF group before, after, and after rest. In the CRS group, however, only the bilateral comparisons before and after the intervention improved significantly. In summary, the range of motion was increased in all groups. The dynamic balance ability improved in SS and PNF groups, and the subacromial space improved in the CRS and PNF.

All muscles of the human body contain various mechanoreceptors that transmit a stimulus to the central nervous system when a stimulus is provided [18]. The muscle spindle and Golgi tendon organ are important mechanoreceptors in the stretching reflex. These two receptors are sensitive to changes in muscle length. The Golgi tendon organs are also affected by changes in muscle tension [19]. Stretching prevents muscle damage, stretches the connective tissue around joints, strengthens exercise performance, and helps to rehabilitate muscles and skeletal systems [20]. Here, when the muscle was stretched, the muscle spindle and the Golgi tendon organ immediately connected the sensory stimulus to the spinal cord. The initial information sent from the muscle spindle provides information to the central nervous system involved in the muscle market [21]. Stimulation from the central nervous system was returned to the muscle, where the muscle reflexively contracts, and the kidney is resisted. The Golgi tendon organ responds to changes in muscle length and increased tension because sensory stimulation is directed toward the central nervous system. Unlike the signal from the muscle spindle, stimulation from the Golgi tendon organ 
causes the relaxation reflex of antagonists. The relaxation reflex acts as a protective mechanism by allowing elongation within a range that does not exceed the elongation limit that damages the muscle fibers [22]. All stretching methods of static, dynamic, and proprioceptive neuromuscular facilitation have been reported to increase range of motion [20]. Therefore, the SS technique and the maintenance-relaxation technique are effective stretching techniques that increase the range of motion of the joint.

The frequency of once a week was reported to be sufficient to maintain flexibility. The frequency of three and five times a week is necessary to increase flexibility. The frequency of three times a week had a better immediate effect than once and six times a week. Moreover, the frequency of three times and six times a week could acquire flexibility more quickly than once a week. To increase the joint range of motion, two-three times a week are required, and it should be continuously performed at least once a week to maintain flexibility [23]. The duration of muscle stretching, and the number of repetitions can be factors that can affect the effectiveness of SS techniques. Applying the SS technique for approximately 30 seconds is most effective rather than a generally applied extension time of approximately 10 seconds for the muscle stretching time [20]. In many studies applying the PNF stretching technique, the duration of contraction of the antagonist muscles was between three and 15 seconds [23]. The recommended optimal time to maintain this stretched position varies from three seconds to 60 seconds. There is currently no data determining the most effective time, but 30 seconds could be better than any number. SS of each muscle should be repeated three to four times sequentially [24]. In this second study, a stretching time of approximately 15 seconds was applied.

The skin stimuli provided by external stimuli can be related to proprioception, and how individuals measure somatosensory signals to control balance may be different (e.g., differences in preferred sensorimotor strategies) [25].
Skin stimulation would be of greater benefit to subjects that depend more on gravity-inertial signals (gravitydependent subjects) than proprioceptive signals (supportdependent subjects). Gravity-dependent subjects adopt exploratory postural behaviors while exhibiting greater sway to obtain information from gravity signals more efficiently [25]. In addition, the restoration of proprioception is accepted as an important determining factor for the completion of rehabilitation because it has been reported in several previous studies that the decreased proprioception in the relevant region is closely related to the deterioration of motor function [26].

Stretching relaxes the complex of actin and myosin, stretches connective tissues, such as muscles, tendons, and ligaments, and induces a permanent increase through changes in the matrix around cells to improve the joint range of motion and increase readiness for body movement, thereby increasing movement efficiency. This can increase or reduce the risk of injury [27, 28]. Stretching methods include SS that induces relaxation of the muscles and joints by maintaining tension. In contrast, the muscles are stretched for a certain period, dynamic stretching using dynamic joint movement and elasticity, and intentional stimulation of the muscle and tendon proprioceptors. Proprioceptive neuromuscular facilitation stretching, which uses the principle of promoting or inhibiting the response of a neuromuscular mechanism, is commonly used to improve flexibility by stretching, relaxation, and muscle contraction [29]. When static, dynamic, and PNF stretching were applied, the dynamic stretching group showed reduced pain induced by delayed muscle pain compared to the other groups, and effectively restored the range of motion and motor function in all stretching groups [30].

Fatigue of lateral rotation of the humerus causes changes in the kinematics of the scapulothoracic joint and the humeral joint, which may affect the muscle mechanics, the size of the subapical space, and the humeral joint kinematics [31]. After the stretching treatment, the 
increased flexibility of the lateral rotator cuff muscles decreased the stiffness of the back of the shoulder, which is believed to decrease the upward glide of the humerus head during shoulder abduction, thereby reducing the activity of the compensatory trapezius muscle [32]. Therefore, it is essential to maintain the normal space under the scapula for efficient functioning of the shoulder joint [33]. An examination of the degree of recovery of the joint range of motion after all treatments confirmed that the hold-relax technique in flexion, vowel, and external rotation had a slightly stronger recovery effect than the group that applied the SS technique.

This study had some limitations. First, the study was conducted with a small number of study subjects, 30 (10 per group). Therefore, it may be difficult to generalize the study results. Second, the force may vary depending on the patient's condition and each treatment period because the force applied during stretching cannot be matched precisely to the maximum muscle contraction given. Third, the study subjects were divided into three groups using a simple random sampling method. Unfortunately, the disadvantage of the simple random sampling method was that the homogeneity between the study groups was not guaranteed. This problem would not have emerged if the number of study subjects had been larger. More research will be needed with more subjects through the same research procedure. Fourth, in the 3.1 group results, there was a significant difference before the intervention during the ultrasound examination, which is very difficult to explain whether there was a significant difference after rest. Hence, the first error could be due to two therapists arranging multiple subjects rather than one therapist performing the examination and intervention. Because an experimental tool was used, mechanical error may occur because one subject was conducting several experiments together. Moreover, the other experiments were also affected by this experiment. Future studies should exclude these cases as much as possible. Finally, more research will be needed because the standardized stretching technique was not compared with other muscles by applying the standardized stretching technique to the same muscle in several studies. Therefore, it is necessary to select an appropriate technique and method according to the experimenter and muscle, and the researcher's attention is required when applying the stretching technique. Hence, continuous research and analysis will be needed to supplement the deficiencies shown in this study.

\section{Conclusion}

This study compared CRS, SS, and PNF and examined their effects on the shoulder function and balance in healthy adults. The results are as follows. First, it was possible to confirm a clear difference in the adduction motions of all groups in the joint range of motion. Second, in the error test, a significant difference could not be confirmed in all values, but a significant difference was confirmed only in the abduction motion. Third, significant differences in reach were confirmed in all directions in the medial, lateral superior, and lateral inferior of the SS and PNF groups. Moreover, after the intervention, a significant difference in the mean value could be confirmed in all groups except the SS group after rest. Significant differences were observed between the CRS, SS, and PNF groups before and after the intervention except after rest. As a result, the application of CRS is as helpful as the existing SS and PNF, and is helpful for joint range of motion improvement, shoulder balance, and subacromial space.

\section{References}

[1] Han J, Waddington G, Adams R, et al. Assessing proprioception: a critical review of methods. J Sport Health Sci. 2016;5(1):80-90.

[2] Lim WS, Shin HS, Kim IS, et al. The effects of scapular 
pattern and hold-relax technique of PNF on the ROM and VAS in frozen shoulder patients. J Kor Phys Ther, 2002;14(1):15-26.

[3] Zimny ML. Mechanoreceptors in articular tissues. Am J Anat 1998;182(1):16-32.

[4] Ercole B, Antonio S, Ann DJ, et al. How much time is required to modify a fascial fibrosis? J Bodyw Mov Ther, 2010;14(4):318-25.

[5] Bandy WD, Irion JM, Briggler M. The effect of static stretch and dynamic range of motion training on the flexibility of the hamstring muscles. J Orthop Sports Phys Ther, 1998;27(4):295-300.

[6] Moreside JM, McGill SM. Improvements in hip flexibility do not transfer to mobility in functional movement patterns. J Strength Cond Res. 2013;27(10):2635-43.

[7] Sady SP, Wortman MV, Blanke D, et al. Flexibility training: ballistic, static or proprioceptive neuromuscular facilitation? Arch Phys Med Rehabil. 1982;63(6):261-3.

[8] Nelson RT. A comparison of the immediate effects of eccentric training vs static stretch on hamstring flexibility in high school and college athletes. N Am J Sports Phys Ther. 2006;1(2):56.

[9] Samson M, Button DC, Chaouachi A, et al. Effects of dynamic and static stretching within general and activity specific warm-up protocols. J Sports Sci Med. 2012;11(2): 279.

[10] Tolliver S, Drew M, Kitts K, et al. Static Stretching and Proprioceptive Neuromuscular Facilitation Stretching within Collegiate Athletes. Int J Res Ex Phys. 2017;12(2): 22-7.

[11] Kusano K, Nishishita S, Nakamura M, et al. Acute effect and time course of extension and internal rotation stretching of the shoulder on infraspinatus muscle hardness. J Shoulder Elbow Surg. 2017;26(10):1782-8.

[12] Fairall RR, Cabell L, Boergers RJ, et al. Acute effects of self-myofascial release and stretching in overhead athletes with GIRD. J Bodyw Mov Ther. 2017;21(3): 648-52.
[13] Behm DG, Bambury A, Cahill F, et al. Effect of acute static stretching on force, balance, reaction time, and movement time. Med Sci Sports Exerc. 2004;36(8): 1397-402.

[14] Wilson, M. The effect of a core stability, m. gluteus medius and proprioceptive exercise program on dynamic postural control in netball players. Doctor's Degree. University of the Free State. 2014

[15] Gorman PP, Butler RJ, Plisky PJ, et al. Upper Quarter Y Balance Test: reliability and performance comparison between genders in active adults. J Strength Cond Res. 2012;26(11):3043-8.

[16] Michener LA, Yesilyaprak SS, Seitz AL, et al. Supraspinatus tendon and subacromial space parameters measured on ultrasonographic imaging in subacromial impingement syndrome. Knee Surg Sports Traumatol Arthrosc. 2015;23(2):363-9.

[17] Asante S, Acquaah K, Oppong-Yeboah B, et al. Comparative joint position error in patients with nonspecific neck disorders and asymptomatic age-matched individuals. S Afr J Physiother. 2019;75(1):1-7.

[18] Condon SM, Hutton RS. Soleus muscle electromyographic activity and ankle dorsiflexion range of motion during four stretching procedures. Physical Therapy. 1987;67(1): 24-30.

[19] Carter AM, Kinzey SJ, Chitwood LF, et al. Proprioceptive Neuromuscular Facilitation Decreases Muscle Activity During the Stretch Reflex in Selected Posterior Thigh Muscles. J Sport Rehabil. 2000;9(4):269-78.

[20] Hahm SC, Kim IB. Effects of static stretching and hold-relax on recovering range of motion and reducing pain of patients with frozen shoulder. J Korean Phys Ther. 2012;19(3):39-47.

[21] D'Ambrogio KJ, Roth GB. Positional release therapy: Assessment \& treatment of musculoskeletal dysfunction. St. Louis, MO: Mosby. 1997.

[22] Mellion MB, Putukian M. Sports medicine secrets. Elsevier Health Sciences. 2003. 
[23] Lee HO, Shin JW. Review of PNF stretching method. PNF \& Mov. 2009;7(4):1-7.

[24] Shellock FG, Prentice WE. Warming-up and stretching for improved physical performance and prevention of sports-related injuries. Sports medicine. 1985;2(4):267-78.

[25] Baige K, Noé F, Bru N, et al. Effects of compression garments on balance control in young healthy active subjects: a hierarchical cluster analysis. Front Hum Neurosci. 2020;14:1-8.

[26] Jeong YW, Lee SY, Seo TH. The Effects of a Shoulder Complex Stabilization Exercise Combined with Upper Extremity Patterns on Ball Control and Ball Speed in Elementary School Baseball Players. PNF \& Mov. 2020;18(3):343-50.

[27] Kim MY, Jun TW, Kim YS, et al. The effects of different stretching duration on strength and flexibility in ballerina. Korean J Phys Educ. 2005;44:399-406.

[28] Woods K, Bishop P, Jones E. Warm-up and stretching in the prevention of muscular injury. Sports medicine. 2007;37(12):1089-99.
[29] Lee SY, Kim JY. The comparison of low intensity eccentric exercise and dynamic stretching on delayed onset muscle soreness. JKAIS. 2012;13(10):4676-85.

[30] Kim SH. Effects of different stretching methods after exercise on delayed onset muscle soreness and exercise performance. Exerc Sci. 2009;18(4):527-38.

[31] Park SJ, Kim SY. The effect of scapular dynamic taping on pain, disability, upper body posture and range of motion in the postoperative shoulder. J Korean Soc Phys Med. 2018;13(4):149-62.

[32] Moon GD, Lim JY, Kim TH, et al. The Effects of Joint Mobilization and Stretching on the Muscle Activity and Internal Rotation of Shoulder Joint in Patients With Impingement Syndrome With Posterior Shoulder Tightness. Phys Ther Korea. 2020;27(1):38-44.

[33] Choo Y. Effects of mobilization with movement combined with exercise (EMWM) on $\mathrm{ADH}, \mathrm{ROM}$ and functional performance in patients with impingement syndrome of the shoulder. J Korean soc integr med. 2019;7(2):153-63. 